\title{
Periodic Solution for Impulsive Cellar Neural Networks with Time-Varying Delays in the Leakage Terms
}

\author{
Bingwen Liu ${ }^{1}$ and Shuhua Gong ${ }^{2}$ \\ ${ }^{1}$ College of Mathematics and Computer Science, Hunan University of Arts and Science, Changde, Hunan 415000, China \\ ${ }^{2}$ College of Mathematics, Physics and Information Engineering, Jiaxing University, Jiaxing, Zhejiang 314001, China
}

Correspondence should be addressed to Shuhua Gong; shuhuagong@yahoo.cn

Received 29 January 2013; Accepted 12 March 2013

Academic Editor: Chuangxia Huang

Copyright (c) 2013 B. Liu and S. Gong. This is an open access article distributed under the Creative Commons Attribution License, which permits unrestricted use, distribution, and reproduction in any medium, provided the original work is properly cited.

\begin{abstract}
This paper is concerned with impulsive cellular neural networks with time-varying delays in leakage terms. Without assuming bounded and monotone conditions on activation functions, we establish sufficient conditions on existence and exponential stability of periodic solutions by using Lyapunov functional method and differential inequality techniques. Our results are complement to some recent ones.
\end{abstract}

\section{Introduction}

It is well known that impulsive differential equations are mathematical apparatus for simulation of process and phenomena observed in control theory, physics, chemistry, population dynamics, biotechnologies, industrial robotics, economics, and so forth [1-3]. Thus, many neural networks with impulses have been studied extensively, and a great deal of literature is focused on the existence and stability of an equilibrium point [4-7]. In [8-10], the authors discussed the existence and global exponential stability of periodic solution of a class of cellular neural networks (CNNs) with impulse. Recently, Wang et al. [11] considered the following CNNs with impulses and leakage delays:

$$
\begin{array}{r}
x_{i}^{\prime}(t)=-a_{i} x_{i}\left(t-\tau_{i}\right)+\sum_{j=1}^{n} \alpha_{i j}(t) f_{j}\left(x_{j}(t)\right) \\
+\sum_{j=1}^{n} \beta_{i j}(t) f_{j}\left(x_{j}\left(t-\sigma_{i j}\right)\right)+I_{i}(t), \\
t \geq 0, \quad t \neq t_{k}, \\
\Delta x_{i}\left(t_{k}\right)=x_{i}\left(t_{k}^{+}\right)-x_{i}\left(t_{k}^{-}\right)=d_{i k} x_{i}\left(t_{k}\right),
\end{array}
$$

where $\Delta x_{i}\left(t_{k}\right)$ are the impulses at moments $t_{k}$ and $t_{1}<t_{2}<$ $\cdots$ is a strictly increasing sequence such that $\lim _{k \rightarrow \infty} t_{k}=$ $+\infty ; a_{i}>0$ and $\tau_{i}>0$ are constants, and $\alpha_{i j}(t), I_{i}(t)$, and $\beta_{i j}(t)$ are continuous periodic functions with period $T$. Suppose that the following conditions are satisfied.

$\left(A_{1}\right)$ There exist constants $L_{j}^{f}, j=1,2, \ldots n$, such that, for any $\alpha, \beta \in R$,

$$
0<\frac{f_{j}(\alpha)-f_{j}(\beta)}{\alpha-\beta}<L_{j}^{f}, \quad \alpha \neq \beta, j=1,2, \ldots n .
$$

$\left(A_{2}\right) f_{i}(0)=0$ and for $i=1,2, \ldots, n$, there exists a constant $0<M_{i}<+\infty$, such that

$$
\left|f_{i}(\alpha)\right| \leq M_{i}, \quad \alpha \in R .
$$

By using the continuation theorem of coincidence degree theory and a suitable degenerate Lyapunov-Krasovskii functional together with model transformation technique, some results were obtained in [11] to guarantee that all solutions of system (1) converge exponentially to a periodic function. However, to the best of our knowledge, few authors have considered the existence and stability of periodic solutions of system (1) without the assumptions $\left(A_{1}\right)$ and $\left(A_{2}\right)$. Thus, it is worthwhile to continue to investigate the convergence behavior of system (1) in this case. In view of the fact that the coefficients and delays in neural networks are usually time varying in the real world, motivated by the above discussions, 
in this paper, we will consider the problem on periodic solution of the following impulsive CNNs with time-varying delays in the leakage terms:

$$
\begin{gathered}
x_{i}^{\prime}(t)=-c_{i}(t) x_{i}\left(t-\eta_{i}(t)\right)+\sum_{j=1}^{n} a_{i j}(t) f_{j}\left(x_{j}(t)\right) \\
+\sum_{j=1}^{n} b_{i j}(t) g_{j}\left(x_{j}\left(t-\tau_{i j}(t)\right)\right)+I_{i}(t), \\
t \geq 0, \quad t \neq t_{k}, \\
\Delta x_{i}\left(t_{k}\right)=x_{i}\left(t_{k}^{+}\right)-x_{i}\left(t_{k}^{-}\right)=d_{i k} x_{i}\left(t_{k}\right),
\end{gathered}
$$

in which $n$ corresponds to the number of units in a neural network, $x_{i}(t)$ corresponds to the state vector of the $i$ th unit at the time $t$, and $c_{i}(t)$ represents the rate with which the $i$ th unit will reset its potential to the resting state in isolation when disconnected from the network and external inputs at the time $t . a_{i j}(t)$ and $b_{i j}(t)$ are the connection weights at the time $t, \eta_{i}(t)$ and $\tau_{i j}(t)$ denote the transmission delays, $I_{i}(t)$ denotes the external bias on the $i$ th unit at the time $t, f_{j}$ and $g_{j}$ are activation functions of signal transmission, $\Delta x_{i}\left(t_{k}\right)$ are the impulses at moments $t_{k}$, and $0 \leq t_{1}<t_{2}<\cdots$ is a strictly increasing sequence such that $\lim _{k \rightarrow \infty} t_{k}=+\infty$, and $i, j=$ $1,2, \ldots, n$. It is obvious that when $f=g$ and $\eta_{i}(t)$ is a constant function, (1) is a special case of (4).

The main purpose of this paper is to give the conditions for the existence and exponential stability of the periodic solutions for system (4). By applying Lyapunov functional method and differential inequality techniques, without assuming $\left(A_{1}\right)$ and $\left(A_{2}\right)$, we derive some new sufficient conditions ensuring the existence, uniqueness, and exponential stability of the periodic solution for system (4), which are new and complement previously known results. Moreover, an example is also provided to illustrate the effectiveness of our results.

Throughout this paper, we assume that the following conditions hold.

$\left(H_{1}\right)$ For $i, j=1,2, \ldots, n, I_{i}, a_{i j}, b_{i j}: R \rightarrow R$ and $c_{i}, \eta_{i}$, $\tau_{i j}: R \rightarrow R^{+}$are continuous periodic functions with period $T>0$, and $t-\eta_{i}(t) \geq 0$ for all $t \geq 0$. In addition, there exist constants $c_{i}^{+}, \eta_{i}^{+}, I_{i}^{+}, \tau_{i}, a_{i j}^{+}, b_{i j}^{+}$, and $\tau_{i j}^{+}$such that

$$
\begin{gathered}
c_{i}^{+}=\sup _{t \in R} c_{i}(t), \quad \eta_{i}^{+}=\sup _{t \in R} \eta_{i}(t), \\
I_{i}^{+}=\sup _{t \in R}\left|I_{i}(t)\right|, \quad a_{i j}^{+}=\sup _{t \in R}\left|a_{i j}(t)\right|, \\
b_{i j}^{+}=\sup _{t \in R}\left|b_{i j}(t)\right|, \quad \tau_{i j}^{+}=\sup _{t \in R} \tau_{i j}(t), \\
\tau_{i}=\max \left\{\eta_{i}^{+}, \max _{j=1,2, \ldots, n} \tau_{j i}^{+}\right\} .
\end{gathered}
$$

$\left(H_{2}\right)$ The sequence of times $t_{k}(k \in N)$ satisfies $t_{k}<t_{k+1}$ and $\lim _{k \rightarrow+\infty} t_{k}=+\infty$, and $d_{i k}$ satisfies $-2 \leq d_{i k} \leq 0$ for $i \in\{1,2, \ldots, n\}$ and $k \in Z^{+}$, where $Z^{+}$denotes the set of all positive integers.
$\left(H_{3}\right)$ There exists a $q \in Z^{+}$such that

$$
d_{i(k+q)}=d_{i k}, \quad t_{k+q}=t_{k}+T, \quad\left(k \in Z^{+}\right) .
$$

$\left(H_{4}\right)$ For each $j \in\{1,2, \ldots, n\}$, there exist nonnegative constants $L_{j}^{f}$ and $L_{j}^{g}$ such that, for all $u, v \in R$,

$$
\begin{gathered}
g_{j}(0)=f_{j}(0)=0, \quad\left|g_{j}(u)-g_{j}(v)\right| \leq L_{j}^{g}|u-v|, \\
\left|f_{j}(u)-f_{j}(v)\right| \leq L_{j}^{f}|u-v| .
\end{gathered}
$$

$\left(H_{5}\right)$ For all $t>0$ and $i \in\{1,2, \ldots, n\}$, there exist constants $\xi_{i}>0$ and $\eta>0$ such that

$$
\begin{aligned}
-\eta> & -\left[c_{i}(t)-c_{i}(t) \eta_{i}(t) c_{i}^{+}\right] \xi_{i} \\
& +\sum_{j=1}^{n}\left(\left|a_{i j}(t)\right|+c_{i}(t) \eta_{i}(t) a_{i j}^{+}\right) L_{j}^{f} \xi_{j} \\
& +\sum_{j=1}^{n}\left(\left|b_{i j}(t)\right|+c_{i}(t) \eta_{i}(t) b_{i j}^{+}\right) L_{j}^{g} \xi_{j} .
\end{aligned}
$$

For convenience, let $x=\left(x_{1}, x_{2}, \ldots, x_{n}\right)^{T} \in R^{n}$, in which " $T$ " denotes the transposition. We define $|x|=$ $\left(\left|x_{1}\right|,\left|x_{2}\right|, \ldots,\left|x_{n}\right|\right)^{T}$ and $\|x\|=\max _{1 \leq i \leq n}\left\{\left|x_{i}\right|\right\}$. As usual in the theory of impulsive differential equations, at the points of discontinuity $t_{k}$ of the solution $t \mapsto\left(x_{1}(t), x_{2}(t), \ldots, x_{n}(t)\right)^{T}$, we assume that $\left(x_{1}(t), x_{2}(t), \ldots, x_{n}(t)\right)^{T} \equiv\left(x_{1}(t-0), x_{2}(t-\right.$ $\left.0), \ldots, x_{n}(t-0)\right)^{T}$. It is clearly that, in general, the derivative $x_{i}^{\prime}\left(t_{k}\right)$ does not exist. On the other hand, according to system (4), there exists the limit $x_{i}^{\prime}\left(t_{k} \mp 0\right)$. In view of the above convention, we assume that $x_{i}^{\prime}\left(t_{k}\right) \equiv x_{i}^{\prime}\left(t_{k}-0\right)$.

The initial conditions associated with (4) are assumed to be of the form

$$
x_{i}(s)=\phi_{i}(s), \quad s \in\left[-\tau_{i}, 0\right], i=1,2, \ldots, n,
$$

where $\phi_{i}(\cdot)$ denotes a real-valued continuous function defined on $\left[-\tau_{i}, 0\right]$.

\section{Preliminary Results}

The following lemmas will be used to prove our main results in Section 3.

Lemma 1. Let $\left(H_{1}\right)-\left(H_{5}\right)$ hold. Suppose that $x(t)=\left(x_{1}(t)\right.$, $\left.x_{2}(t), \ldots, x_{n}(t)\right)^{T}$ is a solution of system (1) with the initial conditions

$$
x_{i}(s)=\varphi_{i}(s), \quad\left|\varphi_{i}(s)\right|<\xi_{i} \frac{\gamma}{\eta}, \quad s \in\left[-\tau_{i}, 0\right],
$$

where $\gamma=1+\max _{i=1,2, \ldots, n}\left\{\left[c_{i}^{+} \eta_{i}^{+}+1\right] I_{i}^{+}\right\}, i=1,2, \ldots, n$. Then

$$
\left|x_{i}(t)\right|<\xi_{i} \frac{\gamma}{\eta}, \quad \forall t \geq 0, \quad i=1,2, \ldots, n .
$$

Proof. Assume that (11) does not hold. From $\left(\mathrm{H}_{2}\right)$, we have

$$
\left|x_{i}\left(t_{k}^{+}\right)\right|=\left|\left(1+d_{i k}\right)\right|\left|x_{i}\left(t_{k}\right)\right| \leq\left|x_{i}\left(t_{k}\right)\right| \text {. }
$$


So, if $\left|x_{i}\left(t_{k}^{+}\right)\right|>\gamma$, then $\left|x_{i}\left(t_{k}\right)\right|>\gamma$. Thus, we may assume that there exist $i \in\{1,2, \ldots, n\}$ and $t_{*} \in\left(t_{k}, t_{k+1}\right)$ such that

$$
\begin{gathered}
\left|x_{i}\left(t_{*}\right)\right|=\xi_{i} \frac{\gamma}{\eta}, \quad\left|x_{j}(t)\right|<\xi_{j} \frac{\gamma}{\eta} \\
\forall t \in\left[-\tau_{i}, t_{*}\right), \quad j=1,2, \ldots, n .
\end{gathered}
$$

According to (4), we get

$$
\begin{aligned}
x_{i}^{\prime}(t)= & -c_{i}(t) x_{i}\left(t-\eta_{i}(t)\right)+\sum_{j=1}^{n} a_{i j}(t) f_{j}\left(x_{j}(t)\right) \\
& +\sum_{j=1}^{n} b_{i j}(t) g_{j}\left(x_{j}\left(t-\tau_{i j}(t)\right)\right)+I_{i}(t) \\
= & -c_{i}(t) x_{i}(t)+c_{i}(t)\left[x_{i}(t)-x_{i}\left(t-\eta_{i}(t)\right)\right] \\
& +\sum_{j=1}^{n} a_{i j}(t) f_{j}\left(x_{j}(t)\right) \\
& +\sum_{j=1}^{n} b_{i j}(t) g_{j}\left(x_{j}\left(t-\tau_{i j}(t)\right)\right)+I_{i}(t) \\
= & -c_{i}(t) x_{i}(t)+c_{i}(t) \int_{t-\eta_{i}(t)}^{t} x_{i}^{\prime}(s) d s \\
& +\sum_{j=1}^{n} a_{i j}(t) f_{j}\left(x_{j}(t)\right) \\
& +\sum_{j=1}^{n} b_{i j}(t) g_{j}\left(x_{j}\left(t-\tau_{i j}(t)\right)\right)+I_{i}(t), \\
& t>0, \quad t \neq t_{k}, \quad i=1,2, \ldots, n .
\end{aligned}
$$

Calculating the upper left derivative of $\left|x_{i}(t)\right|$, together with (13), (14), $\left(H_{5}\right)$, and

$$
\gamma>\left[c_{i}^{+} \eta_{i}^{+}+1\right] I_{i}^{+},
$$

we obtain

$$
\begin{aligned}
0 \leq & D^{-}\left|x_{i}\left(t_{*}\right)\right| \\
\leq & -c_{i}\left(t_{*}\right)\left|x_{i}\left(t_{*}\right)\right|+c_{i}\left(t_{*}\right) \int_{t_{*}-\eta_{i}\left(t_{*}\right)}^{t_{*}}\left|x_{i}^{\prime}(s)\right| d s \\
& +\sum_{j=1}^{n}\left|a_{i j}\left(t_{*}\right) f_{j}\left(x_{j}\left(t_{*}\right)\right)\right| \\
& +\sum_{j=1}^{n}\left|b_{i j}\left(t_{*}\right)\right|\left|g_{j}\left(x_{j}\left(t_{*}-\tau_{i j}\left(t_{*}\right)\right)\right)\right|+\left|I_{i}\left(t_{*}\right)\right|
\end{aligned}
$$

$$
\begin{aligned}
& =-c_{i}\left(t_{*}\right)\left|x_{i}\left(t_{*}\right)\right|+c_{i}\left(t_{*}\right) \\
& \times \int_{t_{*}-\eta_{i}\left(t_{*}\right)}^{t_{*}} \mid-c_{i}(s) x_{i}\left(s-\eta_{i}(s)\right) \\
& +\sum_{j=1}^{n} a_{i j}(s) f_{j}\left(x_{j}(s)\right) \\
& +\sum_{j=1}^{n} b_{i j}(s) g_{j}\left(x_{j}\left(s-\tau_{i j}(s)\right)\right) \\
& +I_{i}(s) \mid d s \\
& +\sum_{j=1}^{n}\left|a_{i j}\left(t_{*}\right) f_{j}\left(x_{j}\left(t_{*}\right)\right)\right| \\
& +\sum_{j=1}^{n}\left|b_{i j}\left(t_{*}\right)\right|\left|g_{j}\left(x_{j}\left(t_{*}-\tau_{i j}\left(t_{*}\right)\right)\right)\right|+\left|I_{i}\left(t_{*}\right)\right| \\
& \leq-\left[c_{i}\left(t_{*}\right)-c_{i}\left(t_{*}\right) \eta_{i}\left(t_{*}\right) c_{i}^{+}\right]\left|x_{i}\left(t_{*}\right)\right| \\
& +\sum_{j=1}^{n}\left(\left|a_{i j}\left(t_{*}\right)\right|+c_{i}\left(t_{*}\right) \eta_{i}\left(t_{*}\right) a_{i j}^{+}\right) L_{j}^{f} \xi_{j} \frac{\gamma}{\eta} \\
& +\sum_{j=1}^{n}\left(\left|b_{i j}\left(t_{*}\right)\right|+c_{i}\left(t_{*}\right) \eta_{i}\left(t_{*}\right) b_{i j}^{+}\right) L_{j}^{g} \xi_{j} \frac{\gamma}{\eta} \\
& +\left[c_{i}^{+} \eta_{i}^{+}+1\right] I_{i}^{+} \\
& =\left\{-\left[c_{i}\left(t_{*}\right)-c_{i}\left(t_{*}\right) \eta_{i}\left(t_{*}\right) c_{i}^{+}\right] \xi_{i}\right. \\
& +\sum_{j=1}^{n}\left(\left|a_{i j}\left(t_{*}\right)\right|+c_{i}\left(t_{*}\right) \eta_{i}\left(t_{*}\right) a_{i j}^{+}\right) L_{j}^{f} \xi_{j} \\
& \left.+\sum_{j=1}^{n}\left(\left|b_{i j}\left(t_{*}\right)\right|+c_{i}\left(t_{*}\right) \eta_{i}\left(t_{*}\right) b_{i j}^{+}\right) L_{j}^{g} \xi_{j}\right\} \frac{\gamma}{\eta} \\
& +\left[c_{i}^{+} \eta_{i}^{+}+1\right] I_{i}^{+} \\
& <-\eta \frac{\gamma}{\eta}+\left[c_{i}^{+} \eta_{i}^{+}+1\right] I_{i}^{+} \\
& <0 \text {. }
\end{aligned}
$$

It is a contradiction and shows that (11) holds. The proof is now completed.

Remark 2. After the conditions $\left(H_{1}\right)-\left(H_{5}\right)$, the solution of system (4) always exists (see $[1,2])$. In view of the boundedness of this solution, from the theory of impulsive differential equations in [1], it follows that the solution of system (4) with initial conditions (10) can be defined on $[0,+\infty)$.

Lemma 3. Suppose that $\left(H_{1}\right)-\left(H_{5}\right)$ are true. Let $x^{*}(t)=$ $\left(x_{1}^{*}(t), x_{2}^{*}(t), \ldots, x_{n}^{*}(t)\right)^{T}$ be the solution of system (4) with 
initial value $\varphi^{*}=\left(\varphi_{1}^{*}(t), \varphi_{2}^{*}(t), \ldots, \varphi_{n}^{*}(t)\right)^{T}$, and let $x(t)=$ $\left(x_{1}(t), x_{2}(t), \ldots, x_{n}(t)\right)^{T}$ be the solution of system (4) with initial value $\varphi=\left(\varphi_{1}(t), \varphi_{2}(t), \ldots, \varphi_{n}(t)\right)^{T}$. Then, there exists a positive constant $\lambda$ such that

$$
x_{i}(t)-x_{i}^{*}(t)=O\left(e^{-\lambda t}\right), \quad i=1,2, \ldots, n .
$$

Proof. Let $y(t)=x(t)-x^{*}(t)$. Then, for $i \in\{1,2, \ldots, n\}$, it is followed by

$$
\begin{array}{r}
y_{i}^{\prime}(t)=-c_{i}(t)\left(x_{i}\left(t-\eta_{i}(t)\right)-x_{i}^{*}\left(t-\eta_{i}(t)\right)\right) \\
+\sum_{j=1}^{n} a_{i j}(t)\left[f_{j}\left(x_{j}(t)\right)-f_{j}\left(x_{j}^{*}(t)\right)\right] \\
+\sum_{j=1}^{n} b_{i j}(t)\left[g_{j}\left(x_{j}\left(t-\tau_{i j}(t)\right)\right)\right. \\
\left.-g_{j}\left(x_{j}^{*}\left(t-\tau_{i j}(t)\right)\right)\right], \\
t \geq 0, \quad t \neq t_{k}, \\
y_{i}\left(t_{k}^{+}\right)=\left(1+d_{i k}\right) y_{i}\left(t_{k}\right), \quad k=1,2, \ldots .
\end{array}
$$

Define continuous functions $\Gamma_{i}(\omega)$ by setting

$$
\begin{aligned}
\Gamma_{i}(\omega)=-\left[c_{i}(t) e^{\omega \eta_{i}(t)}-\omega\right. \\
\left.\quad-c_{i}(t) e^{\omega \eta_{i}(t)} \eta_{i}(t)\left(\omega+c_{i}^{+} e^{\omega \eta_{i}^{+}}\right)\right] \xi_{i} \\
+\sum_{j=1}^{n}\left(\left|a_{i j}(t)\right|+a_{i j}^{+} c_{i}(t) e^{\omega \eta_{i}(t)} \eta_{i}(t)\right) L_{j}^{f} \xi_{j} \\
+\sum_{j=1}^{n}\left(\left|b_{i j}(t)\right| e^{\omega \tau_{i j}(t)}\right. \\
\left.\quad+b_{i j}^{+} c_{i}(t) e^{\omega \eta_{i}(t)} \eta_{i}(t) e^{\omega \tau_{i j}^{+}}\right) L_{j}^{g} \xi_{j}, \\
\omega \geq 0, \quad t \geq 0, \quad i=1,2, \ldots, n .
\end{aligned}
$$

Then

$$
\begin{aligned}
\Gamma_{i}(0)= & -\left[c_{i}(t)-c_{i}(t) \eta_{i}(t) c_{i}^{+}\right] \xi_{i} \\
& +\sum_{j=1}^{n}\left(\left|a_{i j}(t)\right|+a_{i j}^{+} c_{i}(t) \eta_{i}(t)\right) L_{j}^{f} \xi_{j} \\
& +\sum_{j=1}^{n}\left(\left|b_{i j}(t)\right|+b_{i j}^{+} c_{i}(t) \eta_{i}(t)\right) L_{j}^{g} \xi_{j} \\
< & -\eta, \quad t \geq 0, i=1,2, \ldots, n,
\end{aligned}
$$

which, together with the continuity of $\Gamma_{i}(\omega)$, implies that we can choose two positive constants $\lambda$ and $\bar{\eta}$ such that

$$
\begin{aligned}
& -\bar{\eta}>\Gamma_{i}(\lambda) \\
& =-\left[c_{i}(t) e^{\lambda \eta_{i}(t)}-\lambda\right. \\
& \left.-c_{i}(t) e^{\lambda \eta_{i}(t)} \eta_{i}(t)\left(\lambda+c_{i}^{+} e^{\lambda \eta_{i}^{+}}\right)\right] \xi_{i} \\
& +\sum_{j=1}^{n}\left(\left|a_{i j}(t)\right|+a_{i j}^{+} c_{i}(t) e^{\lambda \eta_{i}(t)} \eta_{i}(t)\right) L_{j}^{f} \xi_{j} \\
& +\sum_{j=1}^{n}\left(\left|b_{i j}(t)\right| e^{\lambda \tau_{i j}(t)}+b_{i j}^{+} c_{i}(t) e^{\lambda \eta_{i}(t)}\right. \\
& \left.\times \eta_{i}(t) e^{\lambda \tau_{i j}^{+}}\right) L_{j}^{g} \xi_{j}, \quad t \geq 0, i=1,2, \ldots, n . \\
& Y_{i}(t)=y_{i}(t) e^{\lambda t}, \quad i=1,2, \ldots, n .
\end{aligned}
$$

Then

$$
\begin{aligned}
Y_{i}^{\prime}(t)= & \lambda Y_{i}(t)-c_{i}(t) e^{\lambda t} y_{i}\left(t-\eta_{i}(t)\right) \\
& +e^{\lambda t}\left\{\sum_{j=1}^{n} a_{i j}(t)\left[f_{j}\left(x_{j}(t)\right)-f_{j}\left(x_{j}^{*}(t)\right)\right]\right. \\
& +\sum_{j=1}^{n} b_{i j}(t)\left[g_{j}\left(x_{j}\left(t-\tau_{i j}(t)\right)\right)\right. \\
\left.-g_{j}\left(x_{j}^{*}\left(t-\tau_{i j}(t)\right)\right)\right] & \\
= & \lambda Y_{i}(t)-c_{i}(t) e^{\lambda \eta_{i}(t)} Y_{i}(t) \\
+ & c_{i}(t) e^{\lambda \eta_{i}(t)}\left[Y_{i}(t)-Y_{i}\left(t-\eta_{i}(t)\right)\right] \\
+ & e^{\lambda t}\left\{\sum_{j=1}^{n} a_{i j}(t)\left[f_{j}\left(x_{j}(t)\right)-f_{j}\left(x_{j}^{*}(t)\right)\right]\right. \\
+\sum_{j=1}^{n} b_{i j}(t)\left[g_{j}\left(x_{j}\left(t-\tau_{i j}(t)\right)\right)\right. & \left.\left.-g_{j}\left(x_{j}^{*}\left(t-\tau_{i j}(t)\right)\right)\right]\right\}
\end{aligned}
$$




$$
\begin{aligned}
& =\lambda Y_{i}(t)-c_{i}(t) e^{\lambda \eta_{i}(t)} Y_{i}(t) \\
& +c_{i}(t) e^{\lambda \eta_{i}(t)} \int_{t-\eta_{i}(t)}^{t} Y_{i}^{\prime}(s) d s \\
& +e^{\lambda t}\left\{\sum_{j=1}^{n} a_{i j}(t)\left[f_{j}\left(x_{j}(t)\right)-f_{j}\left(x_{j}^{*}(t)\right)\right]\right. \\
& +\sum_{j=1}^{n} b_{i j}(t)\left[g_{j}\left(x_{j}\left(t-\tau_{i j}(t)\right)\right)\right. \\
& \left.\left.-g_{j}\left(x_{j}^{*}\left(t-\tau_{i j}(t)\right)\right)\right]\right\} \\
& =\lambda Y_{i}(t)-c_{i}(t) e^{\lambda \eta_{i}(t)} Y_{i}(t)+c_{i}(t) e^{\lambda \eta_{i}(t)} \\
& \times \int_{t-\eta_{i}(t)}^{t}\left\{\lambda Y_{i}(s)-c_{i}(s) e^{\lambda s} y_{i}\left(s-\eta_{i}(s)\right)\right. \\
& +e^{\lambda s} \sum_{j=1}^{n} a_{i j}(s)\left[f_{j}\left(x_{j}(s)\right)-f_{j}\left(x_{j}^{*}(s)\right)\right] \\
& +e^{\lambda s} \sum_{j=1}^{n} b_{i j}(s)\left[g_{j}\left(x_{j}\left(s-\tau_{i j}(s)\right)\right)\right. \\
& \left.\left.-g_{j}\left(x_{j}^{*}\left(s-\tau_{i j}(s)\right)\right)\right]\right\} d s \\
& +e^{\lambda t}\left\{\sum_{j=1}^{n} a_{i j}(t)\left[f_{j}\left(x_{j}(t)\right)-f_{j}\left(x_{j}^{*}(t)\right)\right]\right. \\
& +\sum_{j=1}^{n} b_{i j}(t)\left[g_{j}\left(x_{j}\left(t-\tau_{i j}(t)\right)\right)\right. \\
& \left.\left.-g_{j}\left(x_{j}^{*}\left(t-\tau_{i j}(t)\right)\right)\right]\right\} \\
& t \neq t_{k}, \quad i=1,2, \ldots, n,
\end{aligned}
$$$$
\left|Y_{i}\left(t_{k}^{+}\right)\right|=\left|1+d_{i k}\right|\left|Y_{i}\left(t_{k}\right)\right|, \quad i=1,2, \ldots, n .
$$

We define a positive constant $M$ as follows:

$$
M=\max _{1 \leq i \leq n}\left\{\sup _{s \in\left[-\tau_{i}, 0\right]}\left|Y_{i}(s)\right|\right\}
$$

Let $K$ be a positive number such that

$$
\left|Y_{i}(t)\right| \leq M<K \xi_{i} \quad \forall t \in\left[-\tau_{i}, 0\right], i=1,2, \ldots, n
$$

We claim that

$$
\left|Y_{i}(t)\right|<K \xi_{i}, \quad \forall t>0, \quad i=1,2, \ldots, n
$$

Obviously, (27) holds for $t=0$. We first prove that (27) is true for $0<t \leq t_{1}$. Otherwise, there exist $i \in\{1,2, \ldots, n\}$ and $\rho \in\left(0, t_{1}\right]$ such that one of the following two cases must occur;

$$
\begin{array}{rc}
\text { (1) } Y_{i}(\rho)=K \xi_{i}, & \left|Y_{j}(t)\right|<K \xi_{j} \\
\forall t \in[0, \rho), & j=1,2, \ldots, n, \\
\text { (2) } Y_{i}(\rho)=-K \xi_{i}, \quad & \left|Y_{j}(t)\right|<K \xi_{j} \\
\forall t \in[0, \rho), \quad j=1,2, \ldots, n .
\end{array}
$$

Now, we distinguish two cases to finish the proof.

Case (i). If (28) holds. Then, from (21), (23), and $\left(H_{1}\right)-$ $\left(H_{5}\right)$, we have

$$
\begin{aligned}
0 & \leq Y_{i}^{\prime}(\rho) \\
& =\lambda Y_{i}(\rho)-c_{i}(\rho) e^{\lambda \eta_{i}(\rho)} Y_{i}(\rho)+c_{i}(\rho) e^{\lambda \eta_{i}(\rho)}
\end{aligned}
$$

$$
\begin{aligned}
& \times \int_{\rho-\eta_{i}(\rho)}^{\rho}\left\{\lambda Y_{i}(s)-c_{i}(s) e^{\lambda s} y_{i}\left(s-\eta_{i}(s)\right)\right. \\
& +e^{\lambda s} \sum_{j=1}^{n} a_{i j}(s)\left[f_{j}\left(x_{j}(s)\right)-f_{j}\left(x_{j}^{*}(s)\right)\right] \\
& +e^{\lambda s} \sum_{j=1}^{n} b_{i j}(s)\left[g_{j}\left(x_{j}\left(s-\tau_{i j}(s)\right)\right)\right.
\end{aligned}
$$

$$
\left.\left.-g_{j}\left(x_{j}^{*}\left(s-\tau_{i j}(s)\right)\right)\right]\right\} d s
$$




$$
\begin{aligned}
& +e^{\lambda \rho}\left\{\sum_{j=1}^{n} a_{i j}(\rho)\left[f_{j}\left(x_{j}(\rho)\right)-f_{j}\left(x_{j}^{*}(\rho)\right)\right]\right. \\
& +\sum_{j=1}^{n} b_{i j}(\rho)\left[g_{j}\left(x_{j}\left(\rho-\tau_{i j}(\rho)\right)\right)\right. \\
& \left.\left.-g_{j}\left(x_{j}^{*}\left(\rho-\tau_{i j}(\rho)\right)\right)\right]\right\} \\
& \leq \lambda Y_{i}(\rho)-c_{i}(\rho) e^{\lambda \eta_{i}(\rho)} Y_{i}(\rho)+c_{i}(\rho) e^{\lambda \eta_{i}(\rho)} \\
& \times \int_{\rho-\eta_{i}(\rho)}^{\rho}\left\{\lambda Y_{i}(s)+c_{i}^{+} e^{\lambda \eta_{i}(s)}\left|Y_{i}\left(s-\eta_{i}(s)\right)\right|\right. \\
& +\sum_{j=1}^{n} a_{i j}^{+} L_{j}^{f}\left|Y_{j}(s)\right| \\
& \left.+\sum_{j=1}^{n} b_{i j}^{+} L_{j}^{g} e^{\lambda \tau_{i j}(s)}\left|Y_{j}\left(s-\tau_{i j}(s)\right)\right|\right\} d s \\
& +\sum_{j=1}^{n}\left|a_{i j}(\rho)\right| L_{j}^{f}\left|Y_{j}(\rho)\right| \\
& +\sum_{j=1}^{n}\left|b_{i j}(\rho)\right| L_{j}^{g} e^{\lambda \tau_{i j}(\rho)}\left|Y_{j}\left(\rho-\tau_{i j}(\rho)\right)\right| \\
& \leq-\left[c_{i}(\rho) e^{\lambda \eta_{i}(\rho)}-\lambda-c_{i}(\rho) e^{\lambda \eta_{i}(\rho)} \eta_{i}(\rho)\right. \\
& \left.\times\left(\lambda+c_{i}^{+} e^{\lambda \eta_{i}^{+}}\right)\right] K \xi_{i} \\
& +\sum_{j=1}^{n}\left(\left|a_{i j}(\rho)\right|+a_{i j}^{+} c_{i}(\rho) e^{\lambda \eta_{i}(\rho)} \eta_{i}(\rho)\right) L_{j}^{f} K \xi_{j} \\
& +\sum_{j=1}^{n}\left(\left|b_{i j}(\rho)\right| e^{\lambda \tau_{i j}(\rho)}+b_{i j}^{+} c_{i}(\rho) e^{\lambda \eta_{i}(\rho)}\right. \\
& \left.\times \eta_{i}(\rho) e^{\lambda \tau_{i j}^{+}}\right) L_{j}^{g} K \xi_{j} \\
& =\left\{-\left[c_{i}(\rho) e^{\lambda \eta_{i}(\rho)}-\lambda-c_{i}(\rho) e^{\lambda \eta_{i}(\rho)} \eta_{i}(\rho)\right.\right. \\
& \left.\times\left(\lambda+c_{i}^{+} e^{\lambda \eta_{i}^{+}}\right)\right] \xi_{i} \\
& +\sum_{j=1}^{n}\left(\left|a_{i j}(\rho)\right|+a_{i j}^{+} c_{i}(\rho) e^{\lambda \eta_{i}(\rho)} \eta_{i}(\rho)\right) L_{j}^{f} \xi_{j} \\
& +\sum_{j=1}^{n}\left(\left|b_{i j}(\rho)\right| e^{\lambda \tau_{i j}(\rho)}+b_{i j}^{+} c_{i}(\rho) e^{\lambda \eta_{i}(\rho)}\right. \\
& \left.\left.\times \eta_{i}(\rho) e^{\lambda \tau_{i j}^{+}}\right) L_{j}^{g_{j}} \xi_{j}\right\} K
\end{aligned}
$$

Case (ii). If (29) holds. Then, from (21), (23), and $\left(H_{1}\right)-$ $\left(\mathrm{H}_{5}\right)$, we get

$$
\begin{aligned}
& 0 \geq Y_{i}^{\prime}(\rho) \\
& =\lambda Y_{i}(\rho)-c_{i}(\rho) e^{\lambda \eta_{i}(\rho)} Y_{i}(\rho)+c_{i}(\rho) e^{\lambda \eta_{i}(\rho)} \\
& \times \int_{\rho-\eta_{i}(\rho)}^{\rho}\left\{\lambda Y_{i}(s)-c_{i}(s) e^{\lambda s} y_{i}\left(s-\eta_{i}(s)\right)\right. \\
& +e^{\lambda s} \sum_{j=1}^{n} a_{i j}(s)\left[f_{j}\left(x_{j}(s)\right)-f_{j}\left(x_{j}^{*}(s)\right)\right] \\
& +e^{\lambda s} \sum_{j=1}^{n} b_{i j}(s)\left[g_{j}\left(x_{j}\left(s-\tau_{i j}(s)\right)\right)\right. \\
& \left.\left.-g_{j}\left(x_{j}^{*}\left(s-\tau_{i j}(s)\right)\right)\right]\right\} d s \\
& +e^{\lambda \rho}\left\{\sum_{j=1}^{n} a_{i j}(\rho)\left[f_{j}\left(x_{j}(\rho)\right)-f_{j}\left(x_{j}^{*}(\rho)\right)\right]\right. \\
& +\sum_{j=1}^{n} b_{i j}(\rho)\left[g_{j}\left(x_{j}\left(\rho-\tau_{i j}(\rho)\right)\right)\right. \\
& \left.\left.-g_{j}\left(x_{j}^{*}\left(\rho-\tau_{i j}(\rho)\right)\right)\right]\right\} \\
& \geq \lambda Y_{i}(\rho)-c_{i}(\rho) e^{\lambda \eta_{i}(\rho)} Y_{i}(\rho)+c_{i}(\rho) e^{\lambda \eta_{i}(\rho)} \\
& \times \int_{\rho-\eta_{i}(\rho)}^{\rho}\left\{\lambda Y_{i}(s)-c_{i}^{+} e^{\lambda \eta_{i}(s)}\left|Y_{i}\left(s-\eta_{i}(s)\right)\right|\right. \\
& -\sum_{j=1}^{n} a_{i j}^{+} L_{j}^{f}\left|Y_{j}(s)\right| \\
& \left.-\sum_{j=1}^{n} b_{i j}^{+} L_{j}^{g} e^{\lambda \tau_{i j}(s)}\left|Y_{j}\left(s-\tau_{i j}(s)\right)\right|\right\} d s \\
& -\sum_{j=1}^{n}\left|a_{i j}(\rho)\right| L_{j}^{f}\left|Y_{j}(\rho)\right| \\
& -\sum_{j=1}^{n}\left|b_{i j}(\rho)\right| L_{j}^{g} e^{\lambda \tau_{i j}(\rho)}\left|Y_{j}\left(\rho-\tau_{i j}(\rho)\right)\right| \\
& \geq-\left[c_{i}(\rho) e^{\lambda \eta_{i}(\rho)}-\lambda-c_{i}(\rho) e^{\lambda \eta_{i}(\rho)} \eta_{i}(\rho)\right. \\
& \left.\times\left(\lambda+c_{i}^{+} e^{\lambda \eta_{i}^{+}}\right)\right]\left(-K \xi_{i}\right) \\
& +\sum_{j=1}^{n}\left(\left|a_{i j}(\rho)\right|+a_{i j}^{+} c_{i}(\rho) e^{\lambda \eta_{i}(\rho)} \eta_{i}(\rho)\right) L_{j}^{f}\left(-K \xi_{j}\right) \\
& +\sum_{j=1}^{n}\left(\left|b_{i j}(\rho)\right| e^{\lambda \tau_{i j}(\rho)}+b_{i j}^{+} c_{i}(\rho) e^{\lambda \eta_{i}(\rho)}\right.
\end{aligned}
$$

$$
\left.\times \eta_{i}(\rho) e^{\lambda \tau_{i j}^{+}}\right) L_{j}^{g}\left(-K \xi_{j}\right)
$$




$$
\begin{aligned}
& =\left\{-\left[c_{i}(\rho) e^{\lambda \eta_{i}(\rho)}-\lambda-c_{i}(\rho) e^{\lambda \eta_{i}(\rho)}\right.\right. \\
& \left.\times \eta_{i}(\rho)\left(\lambda+c_{i}^{+} e^{\lambda \eta_{i}^{+}}\right)\right] \xi_{i} \\
& +\sum_{j=1}^{n}\left(\left|a_{i j}(\rho)\right|+a_{i j}^{+} c_{i}(\rho) e^{\lambda \eta_{i}(\rho)} \eta_{i}(\rho)\right) L_{j}^{f} \xi_{j} \\
& +\sum_{j=1}^{n}\left(\left|b_{i j}(\rho)\right| e^{\lambda \tau_{i j}(\rho)}+b_{i j}^{+} c_{i}(\rho) e^{\lambda \eta_{i}(\rho)}\right. \\
& \left.\left.\times \eta_{i}(\rho) e^{\lambda \tau_{i j}^{+}}\right) L_{j}^{g_{j}} \xi_{j}\right\}(-K)
\end{aligned}
$$

$>\bar{\eta} K>0$

Therefore, (27) holds for $t \in\left[0, t_{1}\right]$. From (24) and (27), we know that

$$
\begin{array}{r}
\left|Y_{i}\left(t_{1}\right)\right|=\left|y_{i}\left(t_{1}\right)\right| e^{\lambda t_{1}}<K \xi_{i}, \quad i=1,2, \ldots, n, \\
\left|Y_{i}\left(t_{1}^{+}\right)\right|=\left|1+d_{i 1}\right|\left|Y_{i}\left(t_{1}\right)\right| \leq\left|Y_{i}\left(t_{1}\right)\right|<K \xi_{i}, \\
i=1,2, \ldots, n .
\end{array}
$$

Thus, for $t \in\left[t_{1}, t_{2}\right]$, we may repeat the above procedure and obtain

$$
\left|Y_{i}(t)\right|=\left|y_{i}(t)\right| e^{\lambda t}<K \xi_{i}, \quad \forall t \in\left[t_{1}, t_{2}\right], \quad i=1,2, \ldots, n
$$

Further, we have

$$
\left|Y_{i}(t)\right|=\left|y_{i}(t)\right| e^{\lambda t}<K \xi_{i}, \quad \forall t>0, i=1,2, \ldots, n
$$

That is,

$$
\left|x_{i}(t)-x_{i}^{*}(t)\right| \leq K \xi_{i} e^{-\lambda t}, \quad \forall t>0, i=1,2, \ldots, n .
$$

Remark 4. If $x^{*}(t)=\left(x_{1}^{*}(t), x_{2}^{*}(t), \ldots, x_{n}^{*}(t)\right)^{T}$ is the $T$-periodic solution of system (4), it follows from Lemma 3 that $x^{*}(t)$ is globally exponentially stable.

\section{Main Results}

In this section, we will study existence and exponential stability for periodic solutions of system (4).

Theorem 5. Suppose that all conditions in Lemma 3 are satisfied. Then system (4) has exactly one T-periodic solution $x^{*}(t)$. Moreover, $x^{*}(t)$ is globally exponentially stable.

Proof. Let $x(t)=\left(x_{1}(t), x_{2}(t), \ldots, x_{n}(t)\right)^{T}$ be a solution of system (4) with initial conditions (10). By Remark 2, the solution $x(t)$ can be defined for all $t \in[0,+\infty)$. By hypothesis $\left(H_{1}\right)$, we have, for any natural number $h$,

$$
\begin{aligned}
{\left[x_{i}(t+\right.} & (h+1) T)]^{\prime} \\
= & -c_{i}(t) x_{i}\left(t+(h+1) T-\eta_{i}(t)\right) \\
& +\sum_{j=1}^{n} a_{i j}(t) f_{j}\left(x_{j}(t+(h+1) T)\right) \\
& +\sum_{j=1}^{n} b_{i j}(t) g_{j}\left(x_{j}\left(t+(h+1) T-\tau_{i j}(t)\right)\right) \\
& +I_{i}(t), \quad t \neq t_{k}, i=1,2, \ldots, n .
\end{aligned}
$$

Further, by hypothesis of $\left(H_{3}\right)$, we obtain

$$
\begin{aligned}
x_{i}\left(\left(t_{k}+(h+1) T\right)^{+}\right) \\
\quad=x_{i}\left(t_{k+(h+1) q}^{+}\right) \\
=\left(1+d_{i(k+(h+1) q)}\right) x_{i}\left(t_{k+(h+1) q}\right) \\
=\left(1+d_{i k}\right) x_{i}\left(t_{k}+(h+1) T\right), \quad k=1,2, \ldots
\end{aligned}
$$

Thus, for any natural number $h$, we obtain that $x(t+(h+1) T)$ is a solution of system (4) for all $t+(h+1) T \geq 0$. Hence, $x(t+T)$ is also a solution of (4) with initial values

$$
x_{i}(s+T), \quad s \in\left[-\tau_{i}, 0\right], i=1,2, \ldots, n .
$$

Then, by the proof of Lemma 3, there exists a constant $K>0$ such that for any natural number $h$,

$$
\begin{aligned}
& \mid x_{i}(t+(h+1) T)-x_{i}(t+h T) \mid \\
&=\left|x_{i}(t+h T+T)-x_{i}(t+h T)\right| \\
& \leq K \xi_{i} e^{-\lambda(t+h T)} \\
&=K \xi_{i} e^{-\lambda t}\left(\frac{1}{e^{\lambda T}}\right)^{h}, \quad t+h T \geq 0, \\
& \quad t \neq t_{k}, \quad i=1,2, \ldots, n, \\
&\left|x_{i}\left(\left(t_{k}+(h+1) T\right)^{+}\right)-x_{i}\left(\left(t_{k}+h T\right)^{+}\right)\right| \\
& \quad=\left(1+d_{i k}\right)\left|x_{i}\left(t_{k}+(h+1) T\right)-x_{i}\left(t_{k}+h T\right)\right| \\
& \quad \leq K \xi_{i} e^{-\lambda\left(t_{k}+h T\right)} \\
& \quad=K \xi_{i} e^{-\lambda t_{k}}\left(\frac{1}{e^{\lambda T}}\right)^{h}, \quad \forall k \in Z^{+}, i=1,2, \ldots, n .
\end{aligned}
$$


Moreover, for any natural number $m$, we can obtain

$$
\begin{aligned}
& x_{i}(t+(m+1) T) \\
& =x_{i}(t)+\sum_{h=0}^{m}\left[x_{i}(t+(h+1) T)-x_{i}(t+h T)\right], \\
& t+h T \geq 0, \quad t \neq t_{k}, \quad i=1,2, \ldots, n, \\
& \left(x_{i}\left(\left(t_{k}+(m+1) T\right)^{+}\right)\right) \\
& =x_{i}(t)+\sum_{h=0}^{m}\left[x_{i}\left(\left(t_{k}+(h+1) T\right)^{+}\right)\right. \\
& \left.\quad-\left(x_{i}\left(\left(t_{k}+h T\right)^{+}\right)\right)\right],
\end{aligned}
$$

$\forall k \in Z^{+}, \quad i=1,2, \ldots, n$.

Combining (39) with (40), we know that $x(t+m T)$ will converge uniformly to a piecewise continuous function $x^{*}(t)=\left(x_{1}^{*}(t), x_{2}^{*}(t), \ldots, x_{n}^{*}(t)\right)^{T}$ on any compact set of $R$.

Now we are in the position of proving that $x^{*}(t)$ is a $T$-periodic solution of system (4). It is easily known that $x^{*}(t)$ is $T$-periodic since

$$
\begin{aligned}
x_{i}^{*}(t+T) & =\lim _{m \rightarrow+\infty} x_{i}(t+T+m T) \\
& =\lim _{m+1 \rightarrow+\infty} x_{i}(t+(m+1) T) \\
& =x_{i}^{*}(t), \quad t \neq t_{k}, \\
x_{i}^{*}\left(\left(t_{k}+T\right)^{+}\right) & =\lim _{m \rightarrow+\infty} x_{i}\left(\left(t_{k}+T+m T\right)^{+}\right) \\
& =x_{i}^{*}\left(t_{k}^{+}\right), \quad k=1,2, \ldots,
\end{aligned}
$$

where $i=1,2, \ldots, n$. Noting that the right side of (4) is piecewise continuous, together with (36) and (37), we know that $\left\{x_{i}^{\prime}(t+(m+1) T)\right\}$ converges uniformly to a piecewise continuous function on any compact set of $R \backslash\left\{t_{1}, t_{2}, \ldots\right\}$. Therefore, letting $m \rightarrow+\infty$ on both sides of (36) and (37), we get

$$
\begin{aligned}
& x_{i}^{* \prime}(t)=-c_{i}(t) x_{i}^{*}\left(t-\eta_{i}(t)\right) \\
&+\sum_{j=1}^{n} a_{i j}(t) f_{j}\left(x_{j}^{*}(t)\right) \\
&+\sum_{j=1}^{n} b_{i j}(t) g_{j}\left(x_{j}^{*}\left(t-\tau_{i j}(t)\right)\right) \\
&+I_{i}(t), \quad t \neq t_{k}, \quad i=1,2, \ldots, n, \\
& x_{i}^{*}\left(t_{k}^{+}\right)=\left(1+d_{i k}\right) x_{i}^{*}\left(t_{k}\right), \\
& k=1,2, \ldots, \quad i=1,2, \ldots, n .
\end{aligned}
$$

Thus, $x^{*}(t)=\left(x_{1}^{*}(t), x_{2}^{*}(t), \ldots, x_{n}^{*}(t)\right)^{T}$ is a $T$-periodic solution of system (4).

Finally, by Lemma 3, we can prove that $x^{*}(t)$ is globally exponentially stable. This completes the proof.

\section{An Example}

In this section, we give an example to demonstrate the results obtained in the previous sections.

Example 6. Consider the following impulsive cellar neural network consisting of two neurons with time-varying delays in the leakage terms, which is described by

$$
\begin{aligned}
& x_{1}^{\prime}(t)=-3(|\sin \pi t|+1) x_{1}\left(t-\frac{\sin ^{2} \pi t}{1000}\right) \\
& +\frac{1}{16} \cos ^{2} \pi t f_{1}\left(x_{1}(t)\right) \\
& +\frac{1}{16} \sin ^{2} \pi t f_{2}\left(x_{2}(t)\right) \\
& +\frac{1}{16} \sin ^{2} \pi \operatorname{tg}_{1}\left(x_{1}\left(t-\cos ^{2} \pi t\right)\right) \\
& +\frac{1}{16} \sin ^{2} \pi t g_{2}\left(x_{2}\left(t-2 \sin ^{2} \pi t\right)\right) \\
& +100 \cos \pi t \quad t \neq 2 k-1, \\
& x_{2}^{\prime}(t)=-3(|\cos \pi t|+1) x_{1}\left(t-\frac{\sin ^{4} \pi t}{1000}\right) \\
& +\frac{1}{16} \cos ^{3} \pi t f_{1}\left(x_{1}(t)\right) \\
& +\frac{1}{16} \sin ^{3} \pi t f_{2}\left(x_{2}(t)\right) \\
& +\frac{1}{16} \sin ^{3} \pi t g_{1}\left(x_{1}\left(t-\cos ^{2} \pi t\right)\right) \\
& +\frac{1}{16} \sin ^{3} \pi t g_{2}\left(x_{2}\left(t-2 \sin ^{2} \pi t\right)\right) \\
& +100 \sin \pi t \quad t \neq 2 k-1, \\
& x_{i}\left(t_{k}^{+}\right)=\left(1+d_{i k}\right) x_{i}\left(t_{k}\right), \\
& d_{i(2 s)}=-2, \quad d_{i(2 s-1)}=-1, \\
& t_{k}=k, \quad i=1,2, \quad k, s=1,2, \ldots
\end{aligned}
$$

Here, it is assumed that the activation functions are

$$
\begin{aligned}
& g_{1}(x)=g_{2}(x)=x+2 \sin x, \\
& f_{1}(x)=f_{2}(x)=x+3 \sin x .
\end{aligned}
$$

Noting that

$$
\begin{aligned}
\eta_{1}(t)=\frac{\sin ^{2} \pi t}{1000}, & \eta_{2}(t)=\frac{\sin ^{4} \pi t}{1000}, \\
c_{1}(t)=3(|\sin \pi t|+1), & c_{2}(t)=3(|\cos \pi t|+1),
\end{aligned}
$$




$$
\begin{aligned}
& a_{11}(t)=\frac{1}{16} \cos ^{2} \pi t, a_{12}(t)=\frac{1}{16} \sin ^{2} \pi t, \\
& b_{11}(t)=\frac{1}{16} \sin ^{2} \pi t, b_{12}(t)=\frac{1}{16} \sin ^{2} \pi t, \\
& a_{21}(t)=\frac{1}{16} \cos ^{3} \pi t, a_{22}(t)=\frac{1}{16} \sin ^{3} \pi t, \\
& b_{21}(t)=\frac{1}{16} \sin ^{3} \pi t, b_{22}(t)=\frac{1}{16} \sin ^{3} \pi t, \\
& \tau_{11}(t)=\tau_{21}(t)=\cos ^{2} \pi t, \\
& \tau_{12}(t)=\tau_{22}(t)=2 \sin ^{2} \pi t,
\end{aligned}
$$

then we obtain

$$
\begin{aligned}
-\left[c_{i}(t)\right. & \left.-c_{i}(t) \eta_{i}(t) c_{i}^{+}\right] \xi_{i} \\
& +\sum_{j=1}^{2}\left(\left|a_{i j}(t)\right|+c_{i}(t) \eta_{i}(t) a_{i j}^{+}\right) L_{j}^{f} \xi_{j} \\
& +\sum_{j=1}^{2}\left(\left|b_{i j}(t)\right|+c_{i}(t) \eta_{i}(t) b_{i j}^{+}\right) L_{j}^{g} \xi_{j} \\
< & -\left(3-6 \times \frac{1}{1000} \times 6\right) \\
& +2\left(\frac{1}{16}+6 \times \frac{1}{1000} \times \frac{1}{16}\right) \times 3 \\
& +2\left(\frac{1}{16}+6 \times \frac{1}{1000} \times \frac{1}{16}\right) \times 4 \\
< & -1, \quad \xi_{i}=1, i=1,2 .
\end{aligned}
$$

This yields that system (43) satisfies $\left(H_{1}\right)-\left(H_{5}\right)$. Hence, from Theorem 5, system (43) has exactly one 2-periodic solution. Moreover, the 2-periodic solution is globally exponentially stable.

Remark 7. Since $g_{1}(x)=g_{2}(x)=x+2 \sin x, f_{1}(x)=$ $f_{2}(x)=x+3 \sin x$ and CNNs (43) is a very simple form of CNNs with time-varying delays in the leakage terms, it is clear that the conditions $\left(A_{1}\right)$ and $\left(A_{2}\right)$ are not satisfied. Therefore, all the results in [11-19] and the references therein cannot be applicable to system (43) to obtain the existence and exponential stability of the 2-periodic solutions.

\section{Acknowledgments}

The authors would like to express their sincere appreciation to the reviewers for their helpful comments in improving the presentation and quality of the paper. This work was supported by the National Natural Science Foundation of China (Grant no. 11201184), the Natural Scientific Research Fund of Hunan Provincial of China (Grant no. 11JJ6006), the Construct Program of the Key Discipline in Hunan Province (Mechanical Design and Theory), the Natural Scientific Research Fund of Hunan Provincial Education Department of China (Grants nos. 11C0916 and 11C0915), the Natural Scientific Research Fund of Zhejiang Provincial of China (Grant no. LY12A01018), and the Natural Scientific Research Fund of Zhejiang Provincial Education Department of China (Grant no. Z201122436).

\section{References}

[1] V. Lakshmikantham, D. D. Baĭnov, and P. S. Simeonov, Theory of Impulsive Differential Equations, World Scientific, Singapore, 1989.

[2] A. M. Samoilenko and N. A. Perestyuk, Impulsive Differential Equations, World Scientific Publishing, Singapore, 1995.

[3] M. U. Akhmet, "On the general problem of stability for impulsive differential equations," Journal of Mathematical Analysis and Applications, vol. 288, no. 1, pp. 182-196, 2003.

[4] K. Gopalsamy, "Stability of artificial neural networks with impulses," Applied Mathematics and Computation, vol. 154, no. 3, pp. 783-813, 2004.

[5] Z. H. Guan and G. Chen, "On delayed impulsive Hopfield neural networks," Neural Networks, vol. 12, no. 2, pp. 273-280, 1999.

[6] Q. Zhou, "Global exponential stability of BAM neural networks with distributed delays and impulses," Nonlinear Analysis, vol. 10, no. 1, pp. 144-153, 2009.

[7] B. Liu and L. Huang, "Global exponential stability of BAM neural networks with recent-history distributed delays and impulses," Neurocomputing, vol. 69, no. 16-18, pp. 2090-2096, 2006.

[8] Y. Yang and J. Cao, "Stability and periodicity in delayed cellular neural networks with impulsive effects," Nonlinear Analysis, vol. 8, no. 1, pp. 362-374, 2007.

[9] J. Zhang and Z. Gui, "Existence and stability of periodic solutions of high-order Hopfield neural networks with impulses and delays," Journal of Computational and Applied Mathematics, vol. 224, no. 2, pp. 602-613, 2009.

[10] L. Pan and J. Cao, "Anti-periodic solution for delayed cellular neural networks with impulsive effects," Nonlinear Analysis, vol. 12, no. 6, pp. 3014-3027, 2011.

[11] H. Wang, C. Li, and H. Xu, "Existence and global exponential stability of periodic solution of cellular neural networks with impulses and leakage delay," International Journal of Bifurcation and Chaos in Applied Sciences and Engineering, vol. 19, no. 3, pp. 831-842, 2009.

[12] Z. Chen and M. Yang, "Exponential convergence for HRNNs with continuously distributed delays in the leakage terms," Neural Computing and Applications, 2012.

[13] Z. Chen, "A shunting inhibitory cellular neural network with leakage delays and continuously distributed delays of neutral type," Neural Computing and Applications, 2012.

[14] Z. Chen and J. Meng, "Exponential convergence for cellular neural networks with time-varying delays in the leakage terms," Abstract and Applied Analysis, vol. 2012, Article ID 941063, 11 pages, 2012.

[15] S. Lakshmanan, J. H. Park, H. Y. Jung, and P. Balasubramaniam, "Design of state estimator for neural networks with leakage, discrete and distributed delays," Applied Mathematics and Computation, vol. 218, no. 22, pp. 11297-11310, 2012.

[16] Z.-G. Wu, J. H. Park, H. Su, and J. Chu, "Discontinuous Lyapunov functional approach to synchronization of time-delay neural networks using sampled-data," Nonlinear Dynamics, vol. 69, no. 4, pp. 2021-2030, 2012. 
[17] Z.-G. Wu, J. H. Park, H. Su, and J. Chu, "Robust dissipativity analysis of neural networks with time-varying delay and randomly occurring uncertainties," Nonlinear Dynamics, vol. 69, no. 3, pp. 1323-1332, 2012.

[18] H. Zhang, "Existence and stability of almost periodic solutions for CNNs with continuously distributed leakage delays," Neural Computing and Applications, 2013.

[19] H. Zhang and M. Yang, "Global exponential stability of almost periodic solutions for SICNNs with continuously distributed leakage delays," Abstract and Applied Analysis, vol. 2013, Article ID 307981, 14 pages, 2013. 


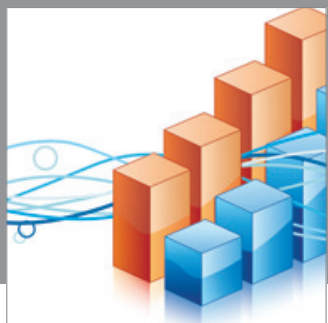

Advances in

Operations Research

mansans

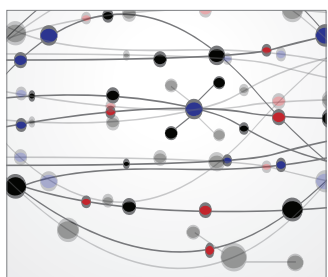

The Scientific World Journal
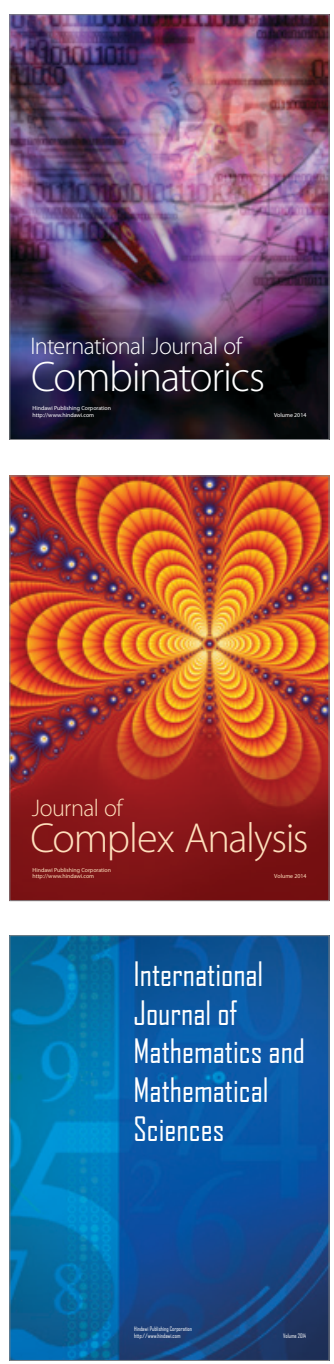
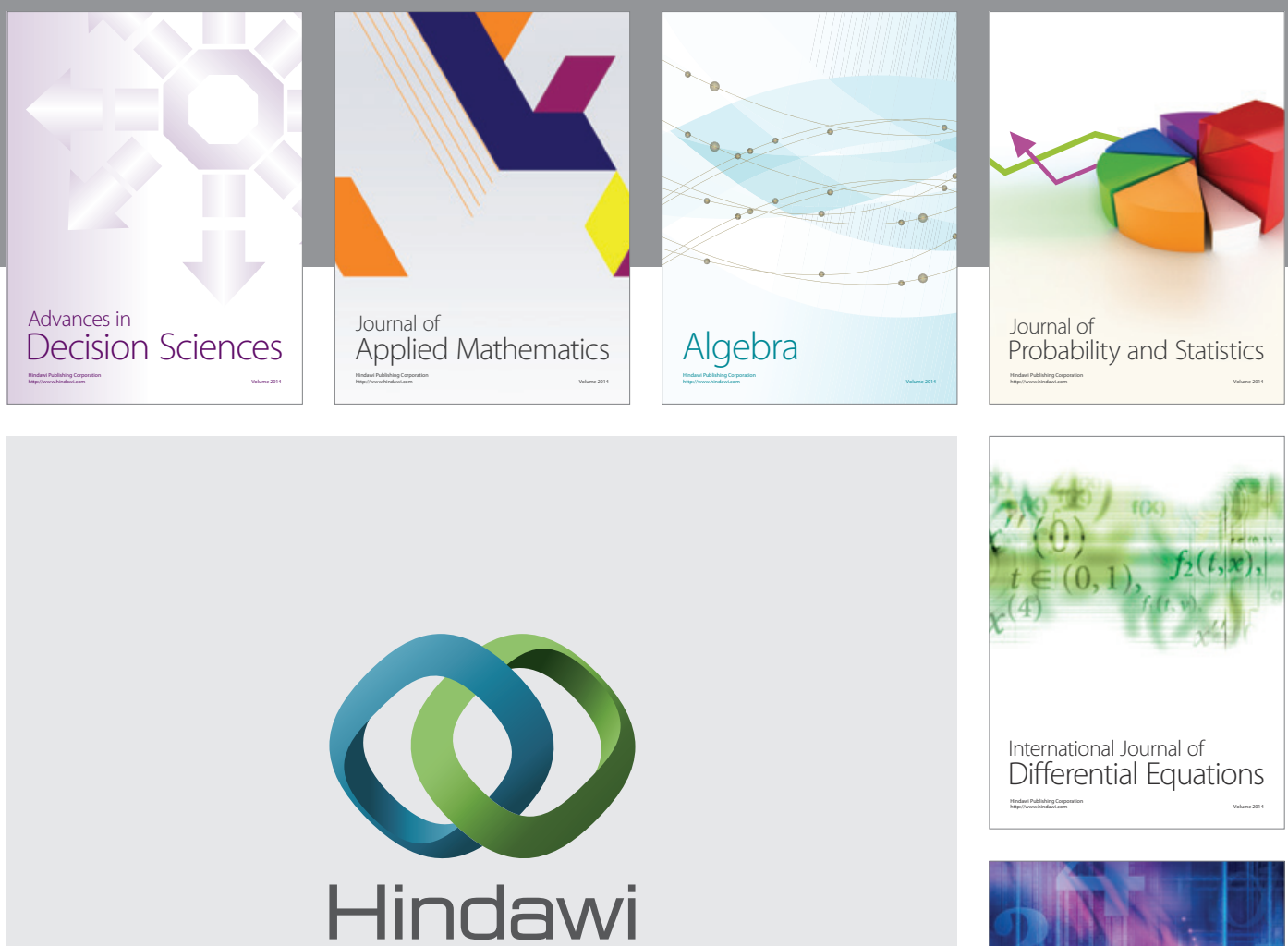

Submit your manuscripts at http://www.hindawi.com
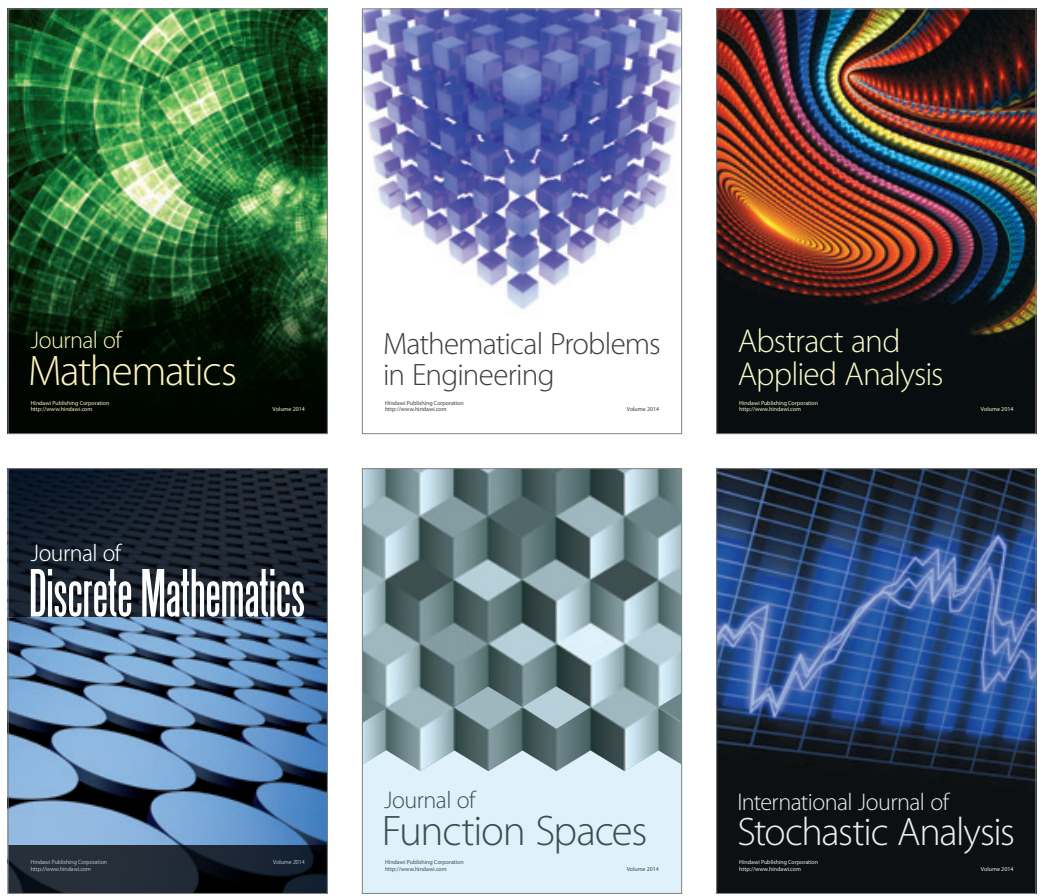

Journal of

Function Spaces

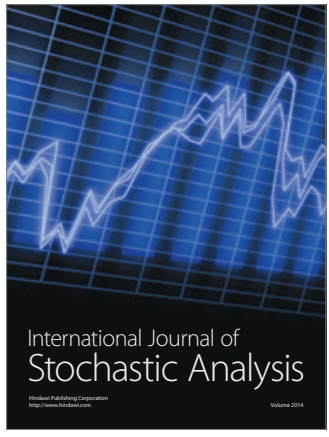

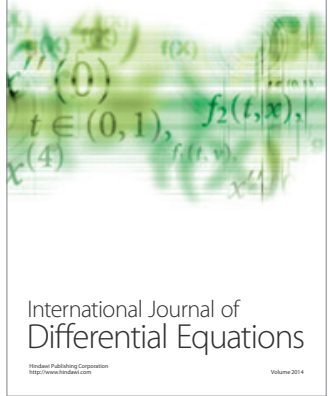
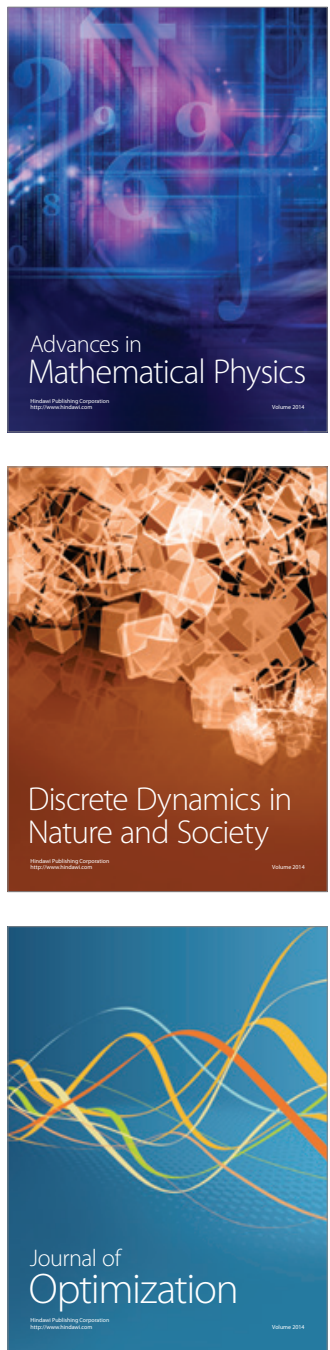